\title{
Acute Hepatitis Due to Dengue Virus in a Chronic Hepatitis Patient
}

Souza L.J ${ }^{1}$, Coelho J.M.C.O. ${ }^{4}$, Silva E.J. ${ }^{2,5}$, Abukater M., ${ }^{1,2}$, Almeida F.C.R. ${ }^{1,2}$, Fonte A. S. ${ }^{1,2}$ and Souza L.A. ${ }^{1,3}$

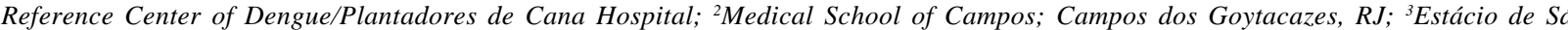
University; ${ }^{4}$ IPEC/FIOCRUZ/RJ; ${ }^{5}$ School of Medical Sciences of State University of Rio de Janeiro/UERJ; Rio de Janeiro, RJ, Brazil

\begin{abstract}
We present a case of acute hepatitis caused by dengue virus, with a significant increase in aspartate transferase and alanine transferase levels in a chronic hepatitis patient attended at the Cane Sugar Planters Hospital of Campos dos Goytacazes, RJ.

Key-Words: Dengue, liver failure, chronic hepatitis.
\end{abstract}

Dengue is an arbovirus disease caused by a flavivirus, with four known serotypes; it is typical of tropical and subtropical regions, where socio-economic conditions favor the development of its vector, Aedes aegypti [1]. Infection by one serotype confers permanent or long-term immunity only against that particular serotype [1,2]. It is believed that more than 100 million inhabitants of tropical countries become infected every year with dengue virus [3].

Dengue can present with a wide spectrum of clinical manifestations, including asymptomatic, classical dengue symptoms, dengue hemorrhagic fever, and atypical forms. The uncommon clinical manifestations of this disease are generally due to a multifactor condition, associated with direct aggression by dengue virus. Dengue can provoke alterations in hepatic functions and typical symptoms of acute hepatitis, such as pain in the right hypochondrium, hepatomegaly, jaundice and elevated transaminase levels. When hepatitis occurs, the transaminase levels reach their maximum on the ninth day after symptoms appear and return to normal within three weeks [4,5].

Laboratory investigation of this arbovirus includes various types of exams, from non-specific analyses to confirmatory serology. Hemograms of patients infected with this virus show leucopenia with atypical lymphocytes, thrombocytopenia $\left(<100,000 / \mathrm{mm}^{3}\right)$ and in some cases, hemoconcentration [1,4]. Low degrees of inflammation and elevated hepatic enzymes are also indicative of infection by dengue.

\section{Case Report}

The patient was 77 years old, male, white, married, retired, born in Campos dos Goytacazes, RJ. Three days previously, the patient began to have fever, anorexia, arthralgia, myalgy, headaches, nausea, vomiting, prostration, a bitter taste in the mouth and a dry cough; he was taking paracetanol. During this period, he also complained of dark "coca cola" urine and light-colored stools. The patient evolved to abdominal extension, associated with pain and feeling full. HPP: Negative Received on 20 May 2008; revised 19 August 2008. Address for correspondence: Dr. Luiz José de Souza. Sociedade Brasileira de Clínica Médica/RJ. Avenida Alberto Torres, 217-Centro. Zip code: 28035-580. Campos dos Goytacazes- Rio de Janeiro- Brazil - Phone/ Fax: (22) 2723-9243.

The Brazilian Journal of Infectious Diseases (c) 2008 by The Brazilian Journal of Infectious Diseases and Contexto Publishing. All rights reserved. for Diabetes Mellitus, high blood pressure, which was being treated with captopril, and a history of dengue fever in 2003. Social status: ex drinker and ex smoker.

\section{Physical Exam}

Awake, in good general condition, normal respiration, not cyanotic, jaundice (+/4), dehydrated (+/4), no fever, normal color. ACV: normal cardiac rhythm in two phases, no audible heart wheezing, blood pressure: 170 x 100mm Hg. AR: no alterations. Abdomen: flaccid, depressible, diffusely painful to deep palpation. Hepatomegaly three $\mathrm{cm}$ from the right costal margin. Spleen not palpable. MMII: without alterations.

Consequently, lab exams were requested.

After seven days, the patient returned to the clinic, presenting strong deterioration of his general condition, with worsening symptoms, and was consequently hospitalized. Physical exam showed prostration, jaundice (4+/4), dehydrated (2+/4), no fever, and normal color. BP: 150 x 110mm Hg. Abdomen: Flaccid, depressible, painful to deep palpation of the hypochondrium and right side. Hepatomegaly $5 \mathrm{~cm}$ from the right costal border. Spleen not palpable.

\section{Complementary Exams}

X-ray of the thorax: Normal. Leucocytes 3,400/uL; Hematocrit: 42\%; hemoglobin 13.9g/dL; hemocytes 5,130 M/ uL; platelets: 95,000/uL; ESR 23mm; AST 8,813 U/L, ALT 3,213 U/L, alkaline phosphatase: 317U/L. Based on these symptoms, the patient was hospitalized.

In the hospital, the patient improved clinically, with increased jaundice. New exams were requested: Leucocytes 7,600/uL, hematocrit 39.4\%, hemoglobin $13.4 \mathrm{~g} / \mathrm{dL}$, red blood cells $4,860 \mathrm{M} / \mathrm{uL}$, platelets 216,000 / uL, AST 1.081U/L, ALT 1.625U/L, TAP 18seg./18/\%, total proteins 6.9 (Alb. 2.2/Glob. 4.7), creatinine 0.92, urea 23.5, total bilirubin $20.12 \mathrm{mg} / \mathrm{dL}$ (BD18.23/BI 1.89), amylase $82 \mathrm{U} / \mathrm{L}$, lipase 50U/L, alkaline phosphatase 290U/L, gamaGT 82U/L. IgM serology for dengue: reactive, antiHAV(IgG): reactive, anti-HAV(IgM), HbsAg, HbeAg, anti-Hbs, anti-Hbc(IgM), anti-HCV, seroagglutination for leptospirosis and serology for yellow fever: Not reactive (Tables 1 and 2).

USG of the abdomen: Echogenic liver, with normal dimensions, suggesting light to moderate steatosis, without other alterations. 
Table 1. Laboratory test results of the dengue patient.

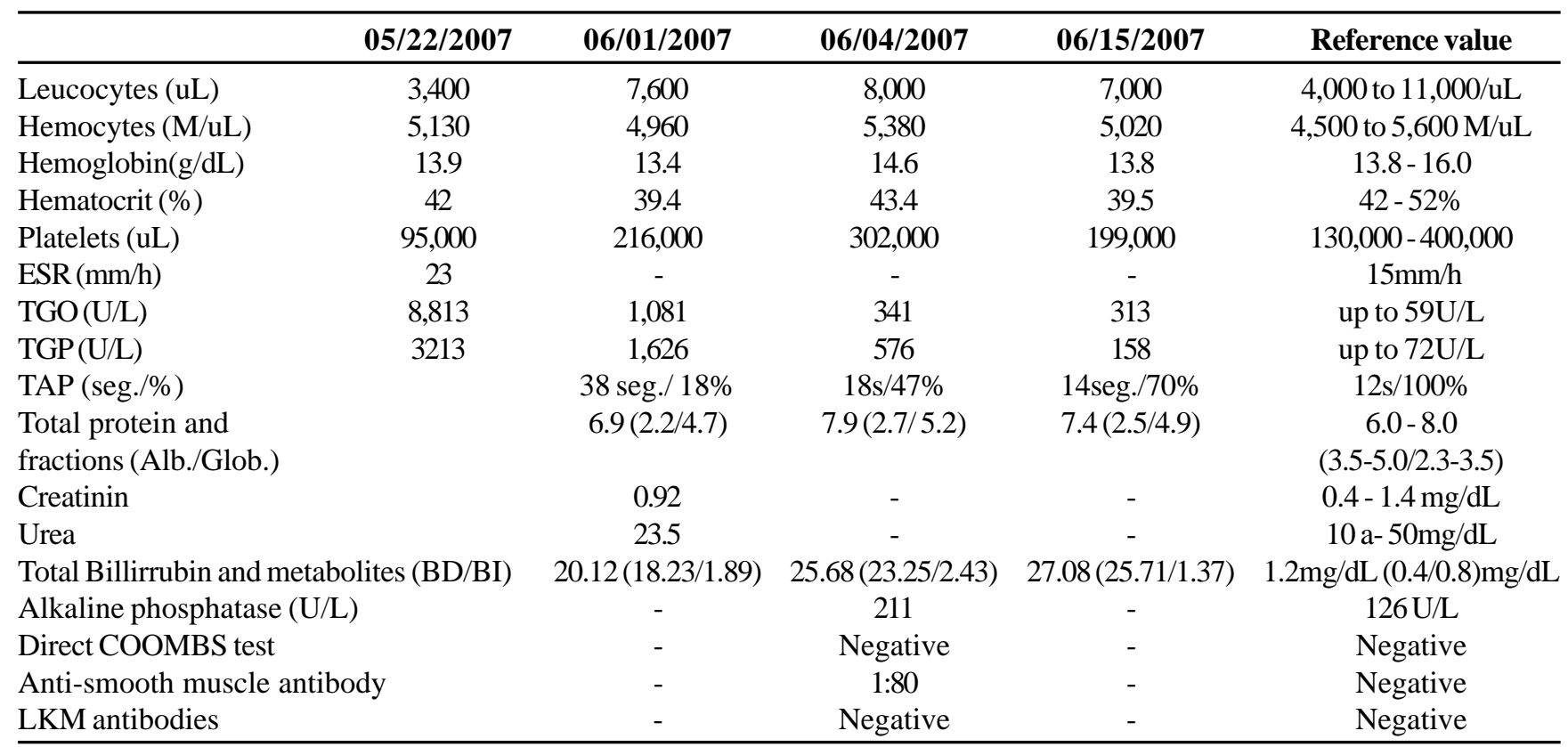

Table 2. Serology information for the dengue patient.

\begin{tabular}{lcc}
\hline & $\mathbf{0 5 / 3 0 / 2 0 0 7}$ & Reference value \\
\hline IgM serology for Dengue & Reactive & - \\
Anti-HAV IgG & Reactive & \\
Anti-HAV IgM & Not reactive & - \\
HbsAg & Not reactive & - \\
HbeAg & Not reactive & - \\
Anti-Hbs & Not reactive & - \\
Anti-Hbc (IgM) & Not reactive & - \\
Anti-HCV & Not reactive & - \\
Seroagglutination for Leptospirosis & Not reactive & - \\
Serology for yellow fever & Not reactive & Up to 110 U/L \\
Amylase & $82 U / L$ & Up to 300 U/L \\
Lipase & $50 U / L$ & Up to 126 U/L \\
Alkaline phosphatase & $290 U / L$ & Up to 78 U/L \\
GamaGT & $82 U / L$ &
\end{tabular}

There was a small accumulation of free liquid was observed in the abdomen (ascites), with no other alterations (Figure 1).

On the seventh day of hospitalization, new exams were made: Leucocytes 8,000/uL, hematocrit $43.4 \%$, red blood cells 5,380M/uL, platelets 302.000, TAP 18s/47\%, total bilirubin 25.68mg/dL (BD 23.25/BI 2.43), AST 341U/L, ALT 576U/L, alkaline phosphatase 211U/L, total protein 7.9 (Alb. 2.7/ Glob. 5.2), direct Coombs: negative, anti-smooth muscle: $1: 80$, anti KLM: negative.

Tomography of the abdomen: Free liquid in the cavity, but without other alterations.

Endoscopy of the upper digestive tract: normal.

During hospitalization, the patient was maintained on symptomatic medication, intravenous hydration, furosemid and spironolactone. On the $15^{\text {th }}$ day of hospitalization, after other diagnoses had been discarded, the patient maintained symptoms of jaundice (4+/4) and a hepatic biopsy was solicited.

\section{Anatomical-Pathological Signs \\ Microscopy}

Hepatic biopsy revealed an approximately 18 portal space ratio. Hepatocytes mainly swollen, presenting reactional alterations and isolated necrosis foci. Portal space widened by fibrosis, with porto-portal bridges, next to other portalcenter bridges. Ductal alterations, cholestasis and moderate lymphocytic and neutrophilic infiltration. The color of the PAS did not reveal anomalous deposits. Masson staining revealed hepatocytic nodules. 
Figure 1. Abdominal ultrasound showing ascites.

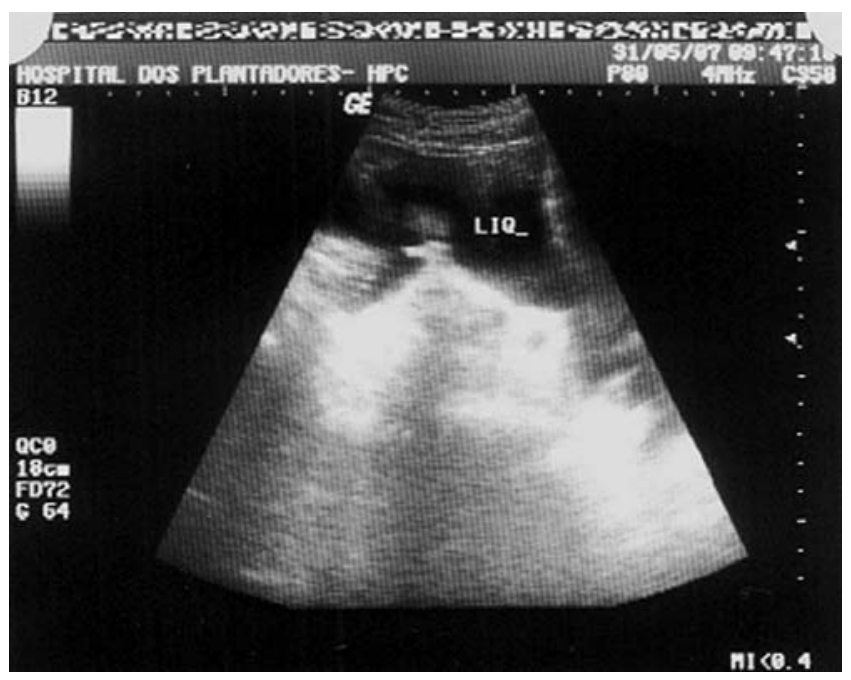

Figure 2. Hepatic biopsy - T. Masson - Hepatic cirrhosis.

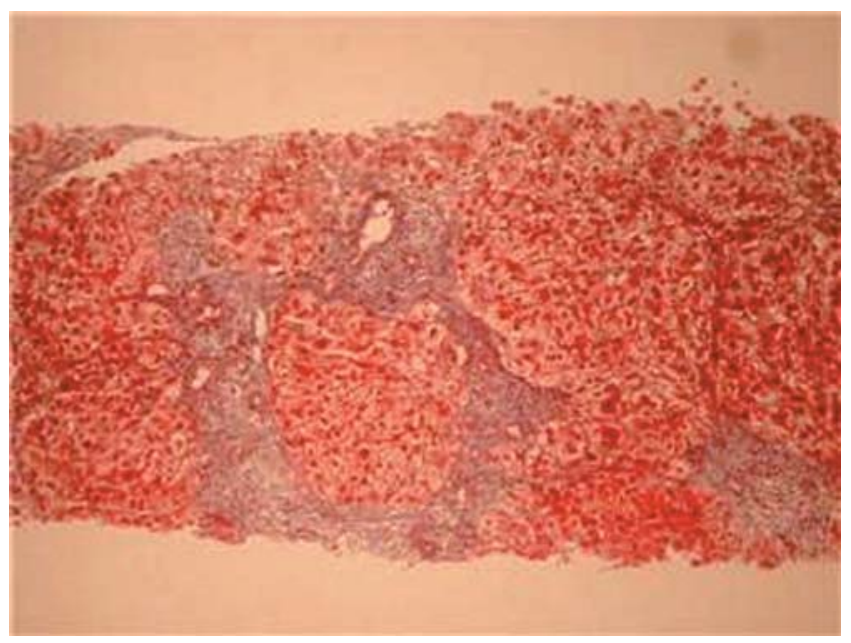

The conclusion about this observation was an acute and chronic hepatopathy associated with hepatic cirrhosis.

Immuno-histochemistry for dengue revealed an immune reaction evident in the cytoplasm of hepatocytes.

The patient was released from the hospital and continued to be treated through ambulatory visits, with remission of symptoms and normalization of hepatic enzymes (Figures 2 and 3).

\section{Discussion}

Dengue is considered the most important arthropodtransmitted virosis that affects man in terms of morbidity and mortality [3]. The world health organization (WHO) estimates that from 50-100 million people are infected annually, in more than 100 countries, on all continents, except Europe [6]. In Brazil, dengue occurs primarily from January to May, because of favorable conditions for the mosquito vector, Aedes

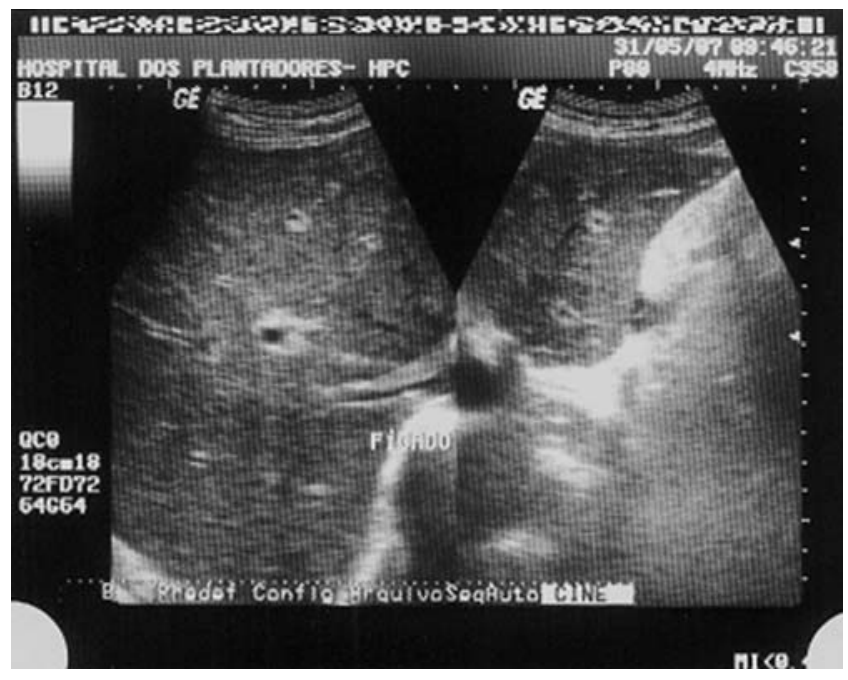

Figure 3. Positive immune reaction for dengue in the cytoplasm of hepatocytes.

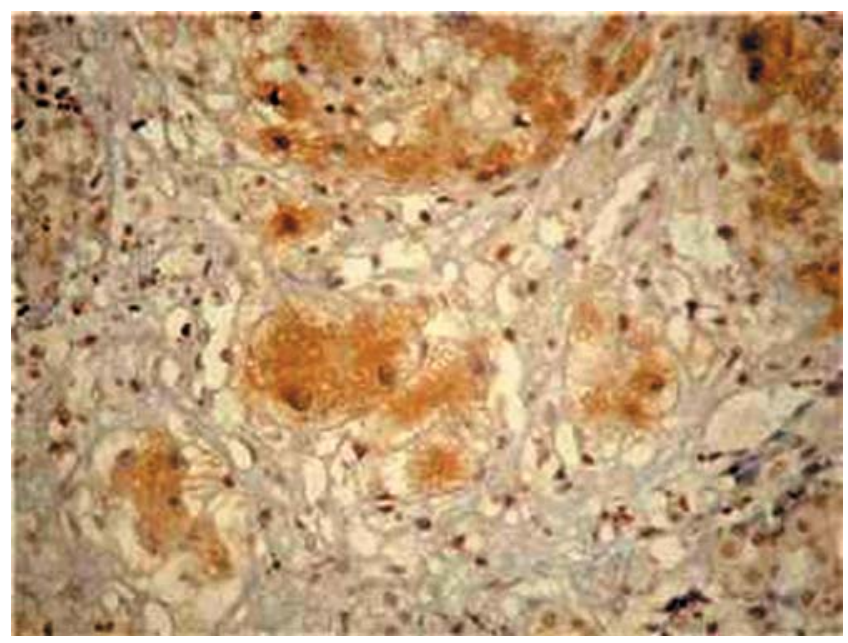

aegypti. During the 1990s, even during non-epidemic years, the disease affected tens of thousands of people per year. The last epidemic in Brazil occurred in 2002, due to the introduction of DEN-3; 794,219 cases were reported, most of them in Rio de Janeiro. In the following years, the dispersion of DEN-3 to other states in Brazil resulted in outbreaks and epidemics, but without reaching the levels of 2002. The secretary for health vigilance of the health ministry (SVS/MS) registered 438,949 cases of classic dengue, 926 cases of hemorrhagic dengue and 98 deaths from January to July 2007 [6]. Consequently, dengue has become one of the principal problems for public health programs.

The clinical picture of classic dengue begins with high fever, headaches, intense myalgy, prostration, nausea, vomiting and arthralgia. The first clinical manifestations of dengue hemorrhagic fever are indistinguishable from those of classic dengue [4]. In some individuals, the clinical 
presentation can assume atypical forms, such as encephalitis, aseptic meningitis, poly neuropathies, acalculous cholecystitis, polyserositis and acute viral hepatitis [4,7]. In most cases of acute hepatitis, caused by dengue virus, the differential diagnosis should include leptospirosis, yellow fever, malaria and hepatitis A, B, and C [4].

The liver is one of the target organs for dengue, and clinical manifestations of hepatic dysfunction can occur during the course of this disease [4]. Hepatic involvement is characterized by acute hepatitis, with pain in the right hypochondrium, hepatomegaly, fever, coagulopathy, increased levels of aminotransferases, and increased bilirubin, leading to jaundice. This can be explained by the host response to the agent, leading to alterations in perfusion in various tissues, in an attempt to preserve vital organs. The liver is deprived of oxygen, leading to lesion of the parenchyma, in which the lesioned hepatocytes liberate transanimases that are detectable in the peripheral blood [1]. In most cases, the high levels of transaminases show the degree of hepatocellular injury, prolonging the clinical course of this disease; however, there is no correlation with prognosis. Another means to evaluate hepatic function is to measure protein synthesis, based on albumin concentration and prothrombin time, which can be measured. Hepatic cirrhosis, characterized by diffuse substitution of the normal hepatic structure by abnormally structured nodules, surrounded by fibrosis, is the common final stage of a series of hepatic pathologies with various causes, such as alchoholism, viral and auto-immune chronic hepatitis, as well as metabolic, vascular and biliary hepatitis [8].

In our patient, the intensity of the alterations observed in the hepatic exams, with initial values of AST 8.813U/L and ALT 3.213U/L, coagulopathy, ascitis and some degree of disorientation, suggested an investigation of contributing pathologies, which lead to a diagnosis of hepatic insufficiency. Initially, drug-induced hepatitis was suggested, because the patient had used paracetanol for several days. Paracetanol can cause liver damage and can lead to or aggravate primary lesions caused by dengue virus. This diagnosis was discarded after hepatic biopsy.

Laboratory and imaging exams excluded a diagnosis of viral hepatitis (A, B and C), auto-immune hepatitis, leptospirosis, biliary tract diseases and obstructive neoplasias. As serology was positive for dengue virus, a hepatic biopsy was made to confirm the diagnosis. Analysis of the liver tissue was made using hematoxilin-eosin, Masson trichrome, PAS, PEARL'S and silver reticulin staining. Observed morphology was that of acute and chronic hepatopathy, associated with liver fibrosis and regeneration nodules, consistent with liver cirrhosis. Immuno-histochemical studies were made, using the streptavidin-biotin-peroxidase technique; a polyclonal antidengue antibody revealed a granular positive immune reaction in the hepatocytes. Consequently, the diagnosis of acute hepatitis due to dengue virus was confirmed in this cirrhotic patient.

\section{References}

1. Souza L.J., Dengue - Diagnóstico Tratamento e Prevenção, Rio de Janeiro: Ed Rúbio, 2007.

2. Wilder-Smith A., Schwartz E. Dengue in Travelers, NEJM 2005;353:924.

3. Focaccia R. (Ed.).Veronesi tratado de infectologia. 3. ed, rev. e atual. São Paulo: Atheneu, 2006. 1 v.

4. Souza L.J., et al. Hepatitis in Dengue Shock Syndrome. Braz J Infect Dis Dec 2002;6(6):322-7.

5. Souza L.J., et al. Aminotransferase changes and acute hepatitis in patients with dengue fever: analysis of 1,585 cases. Braz J Infect Dis Apr 2004;8(2):156-63.

6. Ministério da saúde. Secretaria de Vigilância em Saúde.

7. Nogueira S.A. Dengue: artigo de revisão. Revista de Pediatria Soperj 2001;2(1):22-8.

8. Venancio A.F.A., et al. Cirrose hepática: aspectos morfológicos relacionados às suas possíveis complicações. Um estudo centrado em necropsias. J Bras Patol Med Lab 2005 Feb 41;1.

9. Navarro V.J., Senior J.R. Drug-Related Hepatotoxicity. NEJM 2006;354(16):731.

10. Kasper, Dennis L. (Ed. et al.). Harrison Medicina Interna. $16^{\mathrm{a} e d .}$ Rio de Janeiro: McGraw Hill, c2006. 